NUEVAS NORMAS SOBRE

VALORIZACIÓN DE LOS BIENES DE ACTIVO FIJO: UN RETO A LA INVESTIGACIÓN CONTABLE UNIVERSITARIA

\author{
Mg. ERNESTO POLAR FALCÓN
}

El 15 de Abril de 1998, el Consejo Normativo de Contabilidad, autoridad máxima de la profesión contable en el Perú, ha publicado la Resolución N ${ }^{\circ}$ ()1298-EF/93.01, bajo el título de "Aprueban normas contables referidas a valorización adicional de bienes de activo fijo".

La aplicación de estas normas contables, a partir de 1998 en adelante, por parte de las empresas interesadas en mostrar en sus estados financieros los verdaderos valores de los bienes de sus activos fijos, para otorgar el equilibrio necesario que requiere el Balance General en función de las investigaciones realizadas, motiva a considerarlas como un reto a la investigación contable universitaria, para aportar soluciones y proponer procedimientos de trabajo profesional al Contador Público peruano.

En años anteriores, el llamado "proceso de revaluación voluntaria de activos fijos", tal como era conocido por la profesión contable en el Perú, no alcanzaba sus verdaderos objetivos, porque el Impuesto Extraordinario a los Activos Netos que tenían que pagar las empresas era de carácter confiscatorio. Hoy en día. este impuesto, que actualmente es del $0.5 \%$ del valor de los activos netos, está considerado como "crédito contra el impuesto a la renta", tal como lo expresan textualmente las Leyes $\mathrm{N}^{\circ} 26777,26811$ y 26907 , más el D.S. $N^{\circ}$ 036-EF y la R.S. $N^{\circ}$ 043-98SUNAT,dejando de ser considerado hoy en día como un impuesto directo al patrimonio.

Por estos considerandos urge la necesidad de investigar el contenido de las normas dadas. el entorno empresarial que está interesado en esta valorización adicional de los bienes del activo fijo, los cambios que se abren para culminar este proceso y la evaluación de los resultados que se obtengan, tanto en beneficio de las empresas como del propio Estado, desde el punto de vista financiero y económico.

El significado de la palabra "investigar" lo da el Diccionario de la Real Academia Española de la Lengua en dos acepciones: una primera. que significa "hacer diligencias para descubrir una cosa", y una segunda, consistente en "realizar actividades intelectuales y experimentales de modo sistemático con el propósito de aumentar los conocimientos sobre una determinada materia".

La investigación contable sería entonces, "la actividad intelectual que pretende descubrir las soluciones contables, tributarias, financieras, económicas y societarias adecuadas para los problemas que plantea la valorización adicional de los bienes del activo fijo, tanto a nivel empresarial en general, como a la propia empresa en particular". 
La primera base legal del problema planteado está en el Artículo $228^{\circ}$ de la nueva Ley General de Sociedades-Ley $\mathrm{N}^{\circ}$ 26887-. publicada en el Peruano el 9 de Diciembre de 1997, que textualmente dice lo siguiente:

\section{ARTICULO $228^{\circ}$ - AMORTIZACION Y REVALORIZACION DEL ACTIVO.}

"Los inmuebles. muebles, instalaciones y demás bienes del activo de la sociedad se contabilizan por su valor de adquisición o de costo ajustado por inllación cuando sea aplicable de acuerdo a principios de contabilidad generalmente aceptados en el país. Son amortizados o depreciados anualmente en proporción al tiempo de su vida útil y a la disminución de valor que sufran por su uso o disfrute.

Tales bienes pueden ser objeto de REVALUACION previa comprobación pericial".

Investigar el problema de la valorización adicional de los bienes de activo fijo conduce al investigador a encontrar varios caminos de estudio para buscar soluciones y plantear su aplicación práctica. Uno de estos caminos es estudiar el proceso de preparación de las normas publicadas por el Consejo Normativo de Contabilidad. que ha sido largo, que se inició en Setiembre de 1996 y ha terminado el Abril de 1998; y que debe ser considerado como período positivo de estudio, con la finalidad de emitir normas contables consistentes, dejando a un lado la improvisación y la ligereza que era costumbre. Será interesante investigar los métodos y casos prácticos que han desarrollado los técnicos del Consejo Normativo de Contabilidad. para conocer los objetivos que se propusieron alcanzar, como sustento de las normas emitidas.

Otro de los caminos de investigación es estudiar el área macroeconómica empresarial en la que se van aplicar las normas dadas. Es decir, el estudio de las políticas a seguir por las empresas peruanas, agrupadas por sectores económicos, para modificar las estructuras de sus Balances Generales, con el incremento que se va a mostrar por la valorización adicional de los bienes de activo fijo, que lógicamente va a mejorar los parámetros porcentuales y los índices de los ratios o razones que se apliquen. Se deberá investigar las políticas que adoptarán determinados sectores económicos para emprender campañas de nuevos financiamientos bancarios normales y de la banca de segundo piso, que ofrece interesantes programas de préstamos a largo plazo para la adquisición de equipos y maquinarias. Será interesante investigar las causas que conducen a tomar decisiones de nuevas políticas empresariales a niveles gremiales.

Y por último, otro camino de investigación es estudiar los procedimientos que aplicarán determinadas empresas líderes en sus ramas industriales y comerciales, para incrementar sus bienes de activo fijo por intermedio de las valorizaciones adicionales, como parte de sus programas de expansión a nivel nacional e internacional, que indudablemente traerían nuevas fuentes de trabajo para el pueblo peruano.

Investigar es una tarea seria, propia de personas responsables. ya sean profesionales, docentes universitarios alumnos de las diversas facultades de las universidades peruanas. Se investiga en el campo contable, principalmente nacional, para alcanzar el mayor desarrollo de la contabilidad como ciencia social, que facilite obtener mejor información económica de todos los 
sectores productivos del Perú. Se investiga para que con dicha información económica se incrementen las actividades industriales y comerciales en el país y se multipliquen los puestos de trabajo. Se investiga para que con mayor trabajo, los peruanos alcancen el bienestar necesario y se logren los objetivos nacionales trazados.

Para realizar una investigación con éxito se requieren tres condiciones básicas:

\section{1.- Interesarse al observar un problema}

o fenómeno.- Es necesario que el investigador se interese en los nuevos hechos que se plantean frente al avance de la ciencia contable a nivel mundial y su aplicación en el Perú, así como también frente a la interpretación de nuevas normas contables emitidas por los organismos estatales competentes. En ambos casos, el investigador tiene que estudiar el entorno nacional en el que se van a aplicar. dedicar tiempo a pensar y repensar creativamente, proponiendo alternativas. Unas pueden ser aceptadas y otras rechazadas. El investigador acepta benévolamente los halagos por las alternativas que todos consideran buenas y las críticas por aquellas que son rechazadas; no debe dar muestra de intolerancia y de soberbia, que son incompatibles con la investigación, porque el que cree que lo conoce todo, es incapaz de investigar.

\section{2.- Crear hipótesis y confrontarlas con}

la realidad.- El planteamiento de hipótesis conduce al investigador a desarrollar exigente trabajo para aceptarlas o rechazarlas. Si las comprueba, después de una amplia reflexión, tiene la obligación de confrontarlas con la realidad, obteniendo las conclusiones necesarias y dando las recomendaciones más efectivas para solucionar los problemas.

\section{3.- Perseverancia y capacidad de} concentración.- El investigador realiza esfuerzos que conducen al agotamiento. Es difícil avanzar, ciertamente, por caminos en los que usualmente se encuentran obstáculos. La perseverancia debe ser constante, la concentración en el tema debe ser máxima y la prioridad en el trabajo de investigación sobre otros de carácter profesional o docente, deben ser aceptados por el investigador.

El objetivo de los comentarios vertidos en párrafos anteriores es interesar a los profesionales, docentes y alumnos universitarios de pre grado y post grado. que forman la gran familia contable del Perú, a realizar trabajos de investıgación sobre las diferentes aplicaciones que se presentarán a partir de 1998. en base a las normas dadas en la Resolución $\mathrm{N}^{\circ}$ 012-98-EF/93.01 del Consejo Normativo de Contabilidad, que se presentan a continuación, mostrando al lado izquierdo los considerandos y los artículos de la mencionada Resolución; y al lado derecho las inquietudes de investigación que se plantean como puntos de partida para futuros trabajos de investigación. a corto plazo, ya que muchas empresas van a tomar decisiones de valorización adicional de bienes de activo fijo antes del cierre del ejercicio económico de 1998, para mejorar notoriamente la presentación de sus estados financieros al 31 de diciembre de 1998 


\section{Aprueban normas contables referidas a valorización adicional de bienes de activo fijo.}

\author{
CONSEJO NORMATIVO DE CONTABILIDAD \\ RESOLUCIÓN N 012-98-EF/93.01
}

Lima. 15 de abril de 1998.

(EL PERUANO-Lima, sábado 18 de abril de 1998).

VISTO :

Que, mediante la Resolución No 009-97EF/93.01 del 05 de mayo de 1997, el Consejo Normativo de Contabilidad dictó las normas relativas al tratamiento contable, terminología y otros aspectos de la valorización adicional de los bienes del activo lijo;

Que es necesario compatibilizar la correcta aplicación de las Normas Internacionales de Contabilidad con la Nueva Ley General de Sociedades, y evitar que bajo ninguna forma directa $o$ indirecta se puede distribuir en efectivo u otras formas el excedente de revaluación generado por revaluación voluntaria de los hienes del activo tijo; en tanto esta revaluación no haya sido transferida a Resultados Acumulados o Capital por Aportes tal como lo establece la presente Resolución.

Que el Consejo Normativo de Contabilidad en su vigésima séptima Sesión Ordinaria realizada el 30 de enero de 1998 , consideró necesario modificar y ampliar la Resolución $\mathrm{N}^{\circ}$ 009-97-EF/93.01; acordando que para tal efecto se modifique los Artículos $3^{\circ}, 9^{\circ}$ y $10^{\circ}$ de la referida Resolución; e incluya en las cuentas del Plan Contable General Revisado, las divisionarias necesarias para la aplicación de dicha Resolución : y, asimismo, se establezca que el incremento patrimonial por valorización adicional no pueda ser distribuido en virtud a no constituir una ganancia efectiva de la sociedad;

Que, es necesario una presentación integral de los criterios profesionales a seguir sobre la valorización adicional de los bienes del activo tijo a efecto de lograr la consolidación en una sola Norma todas las actualizaciones sobre la materia;
Resolución $\mathrm{N}^{\circ}$ (0)99-97-EF/93.01 derogada con Art.20 ${ }^{\circ}$ de la presente Resolución.

Investigar compatibilizaciones entre las NICs y la Nueva Leyd e Sociedades.

Investigar contenido de asientos contables relacionados con lavalorización adicional.

Investigar aplicaciones del Plan Contable General Revisado. Investigar la cuenta de valorización adicional como partida no monetaria.

Investigar procesos contables aplicados para consolidarlos en una sola Norma. 
Que, la norma a la que se refiere la presente Resolución está fundada en las normas internacionales de contabilidad, y es de observancia obligatoria para el ejercicio independiente de la profesión de la Contaduría Pública en el país, en resguardo del interés general, y no se opone, por tener el carácter de dictamen profesional, a las disposiciones legales que rijan en el país; $y$,

Estando a lo expuesto, con la aprohación por unanimidad de los integrantes del Consejo Normativo de Contabilidad y en uso de las atribuciones conferidas por los Artículos $2^{\circ}$ y $13^{\circ}$ de la Ley $\mathbf{N}^{\circ} 24680$;

\section{SE RESUELVE:}

Artículo $\mathbf{1}^{\circ}$.- PRECISAR que la valorización adicional de los bienes integrantes de las Cuentas Divisionarias en la Cuenta Principal 33 Inmuebles. Maquinaria y Equipo, del Plan Contable General Revisado, dehe estar sustentada mediante valuación debidamente practicada por profesional competente, en ejercicio independiente; y que el dictamen de valuación debe contener además de la descripción y sustentación de la metodología utilizada, la declaración con el carácter de declaración jurada, que la metodología empleada. fue aplicada con independencia absoluta de criterio profesional: y la estimación de la vida económicamente útil restante del bien valuado que servirá de hase para el cálculo de la depreciación del mayor valor atribuido.

Artículo $\mathbf{2}^{\circ}$.- ADVERTIR. que en ningún caso, la valorización audicional podrá clevar el valor de los bienes del activo tijo por encima del valor que la empresa pueda recuperar, patrimonialmente, del uso o explotación del hien en el futuro mediante la depreciación racional.

Artículo $3^{\circ}$.- INCLUIR, en el listado de las cuentas del Plan Contable General Revisado en la Clase 5 "Patrimonio" en la Cuenta 50. CAPITAL. la cuenta divisionaria 501. Capital por aportes, y 503 . Capital por valorización adicional: en su caso, en la cuenta principal 55. ACCIONARIADO LABORAL, la cuenta divisionaria 551. Accionariado Laboral por aportes y la divisionaria 553. Accionariado Laboral por valorización Adicional; y en
Investigar las relaciones entre las NICs y el dictamen profesional del Contador Público.

Investigar los procesos de valuación de activos fijos que se aplican en el Perú.

Investigar técnicas aplicables a estimar la vida económicamente útil del bien y su relación con la depreciación.

Investigar el proceso aplicable a determinar el valor del bien en función del uso y explotación del mismo.

Investigar dinámicas de las siguientes nuevas divisionarias del P.C.G.R.:

- 503 Capital por Valorización Adicional.

- 553 Accionariado Laboral por

Valorización Adicional. la 
cuenta principal 57. EXCEDENTE DE REVALUACION, la divisionaria 573. Valorización adicional, para el registro del incremento patrimonial por la valorización adicional de los Inmuebles, Maquinaria y Equipo, hasta su real cobertura patrimonial.

Artículo 4 ${ }^{\circ}$.- ESTABLECER. la Cuenta Principal 35 Valorización Adicional de Inmuebles, Maquinaria y Equipo, en la que se debitará en la correspondiente Cuenta Divisionaria, el mayor valor atribuido a los bienes del Activo Fijo, con abono a la Cuenta 573 Valorización Adicional. Esta cuenta podrá tener la apertura a 3 o más dígitos necesarios en concordancia y consistencia con las Cuentas Divisionarias y Analíticas, paralelas de la Cuenta 33 Principal, Inmuebles, Maquinarias y Equipo, que motiva el mayor valor atribuido.

Artículo 5․- ASIGNAR. el Código 32 para el registro oficial de las Provisiones para Desvalorización de los Bienes del Activo Fijo, que en casos especiales hayan sido estaolecidas deliberadamente, de acuerdo con lo facultado en el numeral 1,8 de la Sección I Modalidades Generales del Empleo de las Cuentas del Capítulo II Disposiciones Generales del Plan Contable General Original.

Artículo $6^{\circ}$.- PRECISAR, que el mayor valor atribuido a los bienes del Activo Tijo debe ser registrado en las correspondientes Cuentas Divisionarias y las respectivas Cuentas Analíticas en su caso de la referida Cuenta Frincipal 35, de modo que la Cuenta 33 Inmueoles, Maquinaria y Equipo, sea la que de acuerdo al Principio de Costo Histórico mantenga permanentemente dicho valor. ajustado por inflación; registrando. en su caso, la desvalorización de acuerdo con la regla de los límites de la reexpresión. en la asignada Cuenta 32.

Artículo $7^{\mathbf{}}$.- DETERMINAR. que en todos los casos que se efectúe una revaluación voluntaria. y haya sido establecida; por razones de los límites de reexpresión en años anteriores, provisión para desvalorización de bienes del activo fijo, esta provisión previamente debe ser reverlida, con abono a la Cuenta 765. Devolución de Provisiones de Ejercicios Anteriores.
- 573 Valorización Adicional.

Investigar dinámica de la siguiente Nueva cuenta del P.C.G.R.:

- 35 Valorización Adicional de Inmuebles, Maquinaria y Equipo.

Investigar dinámica de la siguiente Nueva cuenta del P.C.G.R.:

- 32 Provisiones para Desvalorización de los Bienes del Actıvo Fijo.

Investigar aplicación del Principio de Costo Histórico en la Cuenta 33. Investigar aplicación de la nueva Técnica de mayor valor en la Cuenta 35.

Investigar límites contables entre la expresión y la desvalorización de bienes del activo fijo.

Investigar dinámica de la divisionaria.

- 765 Devolución de Provisiones de Ejercicios Anteriores. 
Artículo 8 ${ }^{\circ}$.- PRECISAR. que los ajustes por corrección monetaria deben ser, en contra, 0 a favor; debitados 0 acreditados, respectivamente en la Cuenta 898 REIE ; y, en su caso, las fluctuaciones de menor valor, por desvalorizaciones, que no son generales sino específicas, debe la correspondiente Provisión ser establecida contablemente con cargo a la Cuenta 689 Otras Provisiones del Ejercicio, con excepción de la Desvalorización de Valores y de Existencias, que tienen cuentas establecidas en las divisionarias 683 y 685 respectivamente; independientemente de la adición que se tenga que consignar en la Declaración Jurada Anual para efecto del Impuesto a la Renta..

Artículo $9^{\circ}$.- ESTABLECER. que el incremento patrimonial por valorización adicional retlejada en la contra cuenta 573, Valorización Adicional, no puede ser distribuido como dividendo en efectivo ni en especie y en caso de ser aplicado a cubrir pérdidas, dicho incremento patrimonial deberá ser restituido con la utilidad o utilidades futuras, hasta su total recuperación; supuesto bajo el cual la depreciación del mayor valor se debitará contra los resultados de cada ejercicio anual, efectuándose, en su caso, la adición que corresponda en la Declaración Jurada Anual del Impuesto a la Renta. para conciliar la diferencia temporal que se suscite por la aplicación de los Principios de Contabilidad Generalmente Aceptados y la norma de Impuesto a la Renta.

En caso de ser capitalizado el excedente de revaluación se acreditará a la cuenta 503 . Capital por valorización adicional; $y$, en caso, de que exista Accionariado Laboral, la proporción que le corresponde de dicha capitalización a la cuenta 553. Accionariado Laboral por valorización adicional.

Si después de haber capitalizado el excedente se produce una reducción de Capital, ésra podrá ser hasta el límite del Capital por Aportes, salvo que la reducción de capital sea cancelada mediante adjudicación de los bienes del activo fijo a su valor revaluado.

Artículo $\mathbf{1 0}^{\circ}$.- SEÑALAR, que el incremento patrimonial por valorización adicional será llevado a resultados acumulados o capital por aportes, sólo en el caso de que se realice dicho mayor valor por venta, o por las mayores depreciaciones generadas a partir de la fecha de la Valorización Adicional.
Investigar la carrera de aplicación de los ajustes por inflación en la cuenta 33 con la contrapartida en la divisionaria 898 REIE.

Investigar dinámicas por fluctuaciones de menor valor en las Siguientes divisionarias:

-683 Fluctuación de Valores.

-685 Desvalorización de Existencias

$-689$

Otras Provisiones del Ejercicio.

Investigar la correcta implementaciónde las siguientes dinámicas en el incremento efectuado en la divisionaria 573 - Valorización Adicional: Para cubrir pérdidas o para su capitalización en las divisionarias 503 y 553

Investigar procesos de reducción de capital.

Investigar dinámicas para cancelar reducción de Capital con adjudicación de bienes revaluados del activo fijo.

Investigar las dinámicas aplicables a llevar la valorización adicional a resultados acumulados $o$ a capital por aporte. 
Artículo $11^{\circ}$.- PRECISAR. que las empresas que hayan efectuado revaluaciones voluntarias y éstas hayan cubierto pérdidas o se mantengan en una cuenta del patrimonio, deberán aplicar a partir de la fecha de la presente Resolución, lo dispuesto por los Artículos $9^{\circ}$ y $10^{\circ}$.

Artículo 12 $^{\circ}$.- SENALAR. que cuando el valor en libros de un activo es disminuido como resultado de una subvaluación, la disminución debe ser reconocida como un gasto. Sin embargo, una disminución ulterior a una revaluación voluntaria, debe cargarse directamente contra cualquier excedente de revaluación relacionado en la medida en que la disminución no exceda el monto incluido en el excedente de revaluación con respecto a ese mismo activo, sin perjuicio que éste haya sido capitalizado.

Artículo 13\%.- ACLARAR, que cuando se revalúe una partida de inmuebles, maquinaria y equipo, toda la clase de inmuebles, maquinaria y equipo al cual el activo pertenece debe ser revaluada.

Artículo 14 ${ }^{\circ}$ - SEÑALAR, que el Valor Adicional registrado en la correspondiente Cuenta Divisionaria y, en su caso, Analítica de la Cuenta 35; y el excedente de revaluación acreditado en la cuenta 573, constituyen partidas no Monetarias, provenientes de la Valorización Adicional, que deben ser objeto de aplicación de la Metodología del Ajuste Integral de los Estados Financieros por Efiecto de Inflación establecida en las Resoluciones $\mathrm{N}^{\mathrm{o}} \mathrm{s} .2$ y 3 del Consejo Normativo de Contabilidad.

Artículo $\mathbf{1 5}^{\circ}$.- PRECISAR, que el Ajuste por Corrección Monetaria de los bienes revaluados se debe calcular desde la fecha en que se realizó la revaluación voluntaria que sustenta la valorización voluntaria de los bienes de la Cuenta 33 Inmuebles, Maquinaria y Equipo.

Artículo $16^{\circ}$.- SUPRIMIR, para todos sus efectos la Cuenta Divisionaria 538 Incremento Patrimonial por Valorización ACM, establecida en la Resolución de Contaduría N ${ }^{\circ} 005-94-E F /$ 93.01
Investigar traslado de revaluación voluntarias anteriores con saldos vigentes, a los Art. $9^{\circ}$ y $10^{\circ}$ de la presente Resolución, previa adecuación.

* Investigar dinámicas de subvaluación como gasto o contra un excedente de revaluación en casos específicos.

Investigar la correcta aplicación de la revaluación parcial. cuando ésta forma parte de un total.

Investigar la correcta aplicación de los ajustes por inflación en las siguientes partidas no monetarias: -35 Valorización Adicional de Inmuebles, Maquinaria $\mathrm{y}$ Equipo.

-573 Valorización Adicional.

Investigar la correcta aplicación de los ajustes por inflación desde la fecha de la revaluación voluntaria.

Investigar los problemas que pueden plantearse al suprimir la divisionaria 538 - Incremento Patrimonial por Valorización ACM de la Resolución de Contaduría que aprobó cuentas de ACM en el P.C.G.R. 
Artículo 17 $^{\circ}$.- SUSTITUIR. el Texto del numeral 5 del TITULO INMUEBLES, MAQUINARIA Y EQUIPOS, Subtítulo DEPRECIACION ACUMULADA; PROCEDIMIENTOS DE ACTUALIZACION, incluido por el Art. $8^{\circ}$ de la Resolución $\mathrm{N}^{\circ} 03$ del Consejo Normativo de Contabilidad; el que quedará redactado en la forma siguiente:

"5. En el caso de las Revaluaciones Voluntarias permitidas por la Ley General de Sociedades. de bienes totalmente depreciados, con valor de utilización económica. o con el valor neto en libros inferior al valor de utilización económica. sin perjuicio de cumplir con las disposiciones tributarias sobre el particular. los procedimientos de actualización se aplican desde la fecha de la valorización técnica que sustenta la revaluación voluntaria aprobada por los Organos de decisión de la empresa".

Artículo $18^{\circ}$.- ESTABLECER, que las empresas y entidades que se encuentran bajo el ámbito de supervisión y control de la Superintendencia de Banca y Seguros. se ceñirán a las disposiciones emanadas por dicho Organismo.

Artículo $\mathbf{1 9}^{\circ}$.- DISPONER, que la presente Resolución entre en vigencia el día siguiente de su publicación.

Artículo 20 ${ }^{\circ}$.- DEROGASE, la Resolución $\mathrm{N}^{\circ}$ 009.97.EF/93.01 del Consejo Normativo de Contabilidad.

Regísirese, comuníquese y publíquese.
Investigar el cambio del Numeral 5 del Art. $8^{\circ}$ de la Resolución $\mathrm{N}^{\circ} 3$ del Consejo Normativo de Contabilidad y su aplicación a partir de dicho cambio.

Investigar la implementación de estas normas en el ámbito de supervisión y control de la SBS.

Presente Resolución entra en vigencia al 19 de Abril de 1998.

Sin efecto. 